\title{
Preexposure of MCF-7 breast cancer cell line to dexamethasone alters the cytotoxic effect of paclitaxel but not 5-fluorouracil or epirubicin chemotherapy
}

Breast Cancer - Targets and Therapy

16 March 2017

Number of times this article has been viewed

\author{
Frederic Buxant ${ }^{1, *}$ \\ Nadège Kindt ${ }^{2, *}$ \\ Jean-Christophe Noël ${ }^{3}$ \\ Guy Laurent ${ }^{4, \dagger}$ \\ Sven Saussez ${ }^{2}$
}

'Department of Gynaecology, Iris South Hospital, Brussels, ${ }^{2}$ Department of Anatomy, Laboratory of Anatomy and Cell Biology, Faculty of Medicine and Pharmacy, University of Mons, Mons, ${ }^{3}$ Department of Pathology, Erasme Hospital, Free University of Brussels (ULB), Brussels, ${ }^{4}$ Department of Histology, Laboratory of Histology, Faculty of Medicine and Pharmacy, University of Mons, Mons, Belgium

*These authors contributed equally to this work

tDr Guy Laurent passed away on August 9, 2016
Correspondence: Frederic Buxant Department of Gynaecology, HISIxelles Hospital, Rue Jean Paquot 63, 1050 Brussels, Belgium

$\mathrm{Tel}+3226414134$

Fax +32 26398909

Email fbuxant@his-izz.be
Purpose: Glucocorticoids (GCs) are often administered prior to any chemotherapeutics to prevent the secondary effects of anticancer agents. Glucocorticoid receptors (GRs) are expressed in several types of cancer cells, particularly in several histological types of breast cancer. Activation of GRs is not associated with any specific cellular response. Both proapoptotic and antiapoptotic responses have been observed, depending on the study or the type of breast cancer cells. Therefore, it is of relevance to investigate the possible modulation of apoptotic effect of chemotherapeutic agents when cancerous cells have previously been exposed to GCs.

Methods: In vitro cell growth was assayed by counting MCF-7 cells upon exposure to epirubicin (25 nM), 5-fluorouracil (5-FU) $(15 \mu \mathrm{M})$, and paclitaxel $(15 \mathrm{nM})$, either with or without prior exposure to the GC dexamethasone (Dex) (100 nM).

Results: Following preexposure to Dex, the antiapoptotic activity of paclitaxel was significantly reduced by $8.5 \%(p<0.05)$, but the activities of epirubicin and 5 -FU remained unaltered.

Conclusion: In light of the finding that the response of MCF-7 cells pretreated with Dex was significantly reduced, we recommend that the function of GCs should be defined more precisely if they are to be used in conjunction with chemotherapy.

Keywords: dexamethasone, 5-fluorouracil, epirubicin, paclitaxel, MCF-7, chemotherapy

\section{Introduction}

Glucocorticoids (GCs) are steroid hormones that are critically involved in regulating and resolving inflammatory processes in mammals. They are also involved in many other essential processes, including cellular metabolism, differentiation, apoptosis, and immune response. ${ }^{1}$ Clinically, GCs are used to treat allergic reactions and inflammatory or autoimmune diseases, to reduce soft tissue edema after solid organ transplantation, and to eliminate malignant lymphoid cells by triggering apoptotic cell death. ${ }^{2}$ Various doses of GCs, most commonly dexamethasone (Dex), are frequently administered throughout the course of chemotherapeutic treatment for solid tumors in order to reduce toxicity and to protect normal tissue from the negative effects of continued exposure to genotoxic drugs. Dex is particularly effective in preventing chemotherapy-related hyperemesis. ${ }^{3-5}$ First-line chemotherapy for early and advanced stage breast cancers is based mainly on anthracyclines (doxorubicin or epirubicin), cyclophosphamide, 5-fluorouracil (5-FU) (Merck, Darmstadt, Germany), and taxanes (primarily paclitaxel). ${ }^{6,7}$ Taxanes, such as paclitaxel, are among the most common chemotherapeutic agents currently used for the treatment of breast cancer. Use of taxanes was initially 
limited because of hypersensitivity reactions, but once these could be adequately managed, largely by premedication with GC, taxane chemotherapy became part of the standard breast cancer treatment in most Western countries. ${ }^{4}$

Glucocorticoid receptors (GRs) have been identified in several types of cancerous cells, including breast cancer cell lines such as MCF-7. ${ }^{8-11}$ Exposure of these receptors - functional in MCF-7 cells - to Dex has been reported to inhibit cell proliferation. ${ }^{12}$ Other in vitro studies have shown that GCs inhibit the growth of estrogen receptor (ER) $\alpha$-positive (ER $\alpha$-positive) (eg, MCF-7) cells by arresting cell cycle in G0/G1 phase. In contrast, proliferation of ER $\alpha$-negative (eg, MDA-MB-231) cells is not inhibited by treatment with GCs, suggesting that GCs inhibit the proliferation of breast cancer cells via the ER $\alpha$ signaling pathway. ${ }^{13} \mathrm{GCs}$ and mineralocorticoids can also cross talk with progesterone receptors to induce a progesterone-like effect in breast cancer. ${ }^{14}$ However, Dex treatment of MCF-7 cells has also been reported to promote cell proliferation by upregulating c-Myc, which is induced by the promotion of NF $\kappa$ B transcriptional activity. ${ }^{12,15}$ Loss of GR activation has been observed in BRCA1-mutated breast tissue. ${ }^{9,11}$ While the presence of GRs in specific breast cancer cell lines has been clearly established, their activation is associated with multiple and opposite effects. GCs exert an antiapoptotic effect. This antiapoptotic effect was studied further and could be mediated by the induction of the expression of other genes frequently associated with protection from cell apoptosis, such as Bcl-XL, BAk, SGK-1, and MKP-1. Concomitantly, Dex also reinforces its survival effect by downregulation of proapoptotic genes. ${ }^{16-18}$ Dex induces the expression of genes associated with protection against cell apoptosis. GRs disrupt p53-mediated regulation of cell survival. Loss of p53 activity has been linked with a range of human cancers. P53 mediates cell apoptosis in case of DNA damage or hypoxia. ${ }^{19}$

The majority of chemotherapy patients receive a preadministration of Dex. We deemed that this sequential treatment (ie, administration of Dex followed by a chemotherapeutic agent) warranted further assessment. The present study aimed to investigate what influence Dex treatment, prior to the administration of a chemotherapeutic agent (5-FU, epirubicin, or paclitaxel), had on the proliferation of MCF-7 cells.

\section{Materials and methods}

\section{Cell culture}

MCF-7 cells obtained from (ATCC Bioresource Centre, Manassas, VA, USA), were maintained at $37^{\circ} \mathrm{C}$ in a humidified cell incubator with a $5 \% \mathrm{CO}_{2}$ atmosphere. Cells were cultured in Dulbecco's Modified Essential Medium (DMEM) containing phenol red and supplemented with $10 \%$ fetal bovine serum (FBS; Life Technologies, Paisley, UK), 2 mM L-glutamine, and 1\% penicillin-streptomycin (all from Life Technologies). For the experimental procedures, cells were seeded in DMEM (phenol red-free) supplemented with $10 \%$ charcoal-stripped FBS and $100 \mathrm{nM} 17 \beta$-estradiol.

\section{Measurement of cell culture growth by cell counting}

MCF-7 cells were plated at a density of 15,000 cells $/ \mathrm{mL}$ in 12 -well dishes and then incubated at $37^{\circ} \mathrm{C}$ with a $5 \%$ $\mathrm{CO}_{2}$ atmosphere. The following day, the cell cultures were given fresh medium, with or without Dex (control [CT]), at a concentration of $100 \mathrm{nM}$ for $30 \mathrm{~min}$. The cells were then incubated for 4 hours in a medium containing $15 \mathrm{nM}$ paclitaxel (Sigma-Aldrich, St. Louis, MO, USA) or $15 \mu \mathrm{M} 5$-FU (Merck). Cells were treated with epirubicin hydrochloride (Tocris Bioscience, Bristol, UK) by culturing the cells for 3 days in the presence of $25 \mathrm{nM}$ of the drug. Measurement of cell proliferation was performed 3 days after the drug treatments. Cells were dislodged from the surface of the culture vessel by treatment with $1 \mathrm{~mL}$ of trypsin-EDTA solution (Sigma-Aldrich) for $2 \mathrm{~min}$. After vigorous pipetting, the number of cells in the suspension was determined using an electronic cell counter (model Z1 Coulter counter; Beckman Coulter, Fullerton, CA, USA). The cell counter did not count nonviable cells and only recorded the number of live cells. An equivalent number of cells were counted for each experiment regardless of the treatment conditions.

\section{Statistical analysis}

SigmaPlot $^{\circledR} 11$ software (Systat Software Inc., Chicago, IL, USA) was used for the statistical analyses. Parametric analysis was performed by Student's $t$-test. A $p$-value $<0.05$ was considered statistically significant.

\section{Results}

Cell numbers decreased by more than half $(p<0.001)$ when we incubated MCF-7 with $100 \mathrm{nM}$ Dex for $30 \mathrm{~min}$ compared to untreated (CT) cells (Figure 1). No significant difference was seen between the samples exposed to epirubicin or to Dex and then epirubicin (Figure 2). This was also the case for the samples treated with 5-FU or Dex followed by 5-FU (Figure 3 ). By contrast, a statistically significant difference ( $p=0.038)$ was noted between samples treated with paclitaxel or with Dex followed by paclitaxel (Figure 4). Preexposure of MCF-7 cells to Dex before paclitaxel treatment reduced the cytotoxic effect of paclitaxel by $8.5 \%$. 


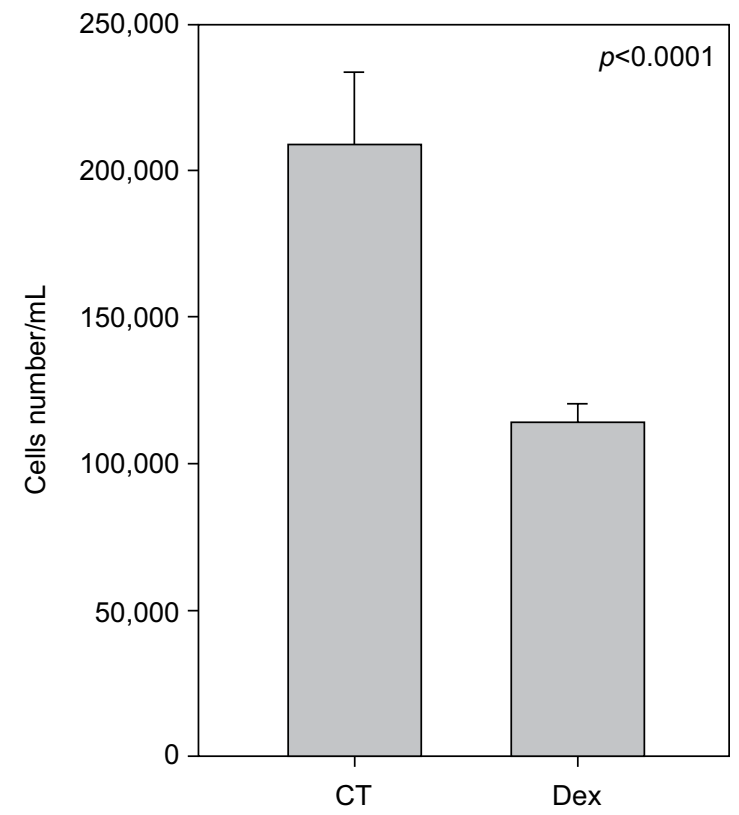

Figure I Untreated and dexamethasone (Dex) controls. MCF-7 cells were either untreated (control [CT]) or treated with $100 \mathrm{nM}$ of Dex. The graph indicates the number of cells $/ \mathrm{mL} 3$ days after treatment.

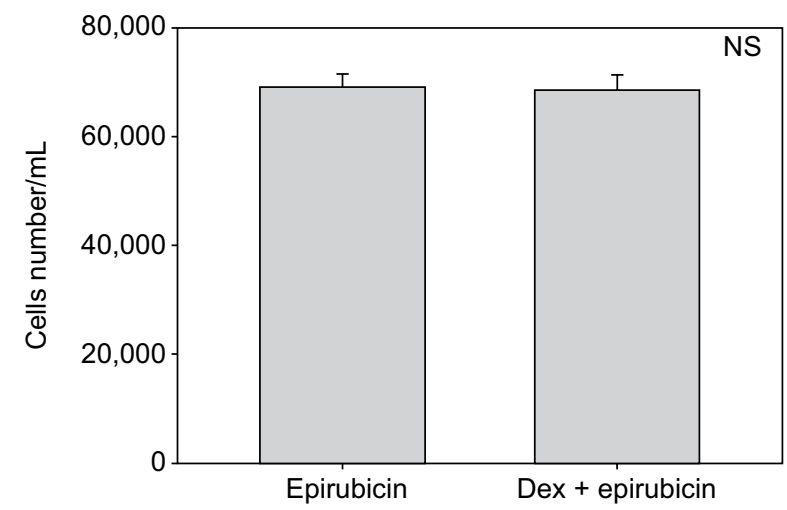

Figure 2 Epirubicin with dexamethasone (Dex) pretreatment. MCF-7 cells were pretreated with $100 \mathrm{nM}$ of Dex prior to administration of $25 \mathrm{nM}$ of epirubicin. The graph indicates the number of cells $/ \mathrm{mL} 3$ days after treatment. No significant difference was discernible between the samples treated with Dex alone or Dex in combination with epirubicin $(n=4)$. Error bars indicate standard deviation.

Abbreviation: NS, nonsignificant.

\section{Discussion}

GCs have been reported to have multiple modes of action, thus explaining their proapoptotic and antiapoptotic effects on ER-positive or ER-negative breast cancer cells, respectively. As GCs are administered systematically with epirubicin- and paclitaxel-based chemotherapies, the consequences of GC exposure in terms of the chemotherapeutic effect warranted further investigation.

We chose to study the apoptotic effects of epirubicin, 5-FU, and paclitaxel when MCF-7 cells were preexposed to Dex because they are the three main drugs currently used in routine chemotherapy. Since the anthracyclines doxorubicin and epirubicin have similar mechanisms of

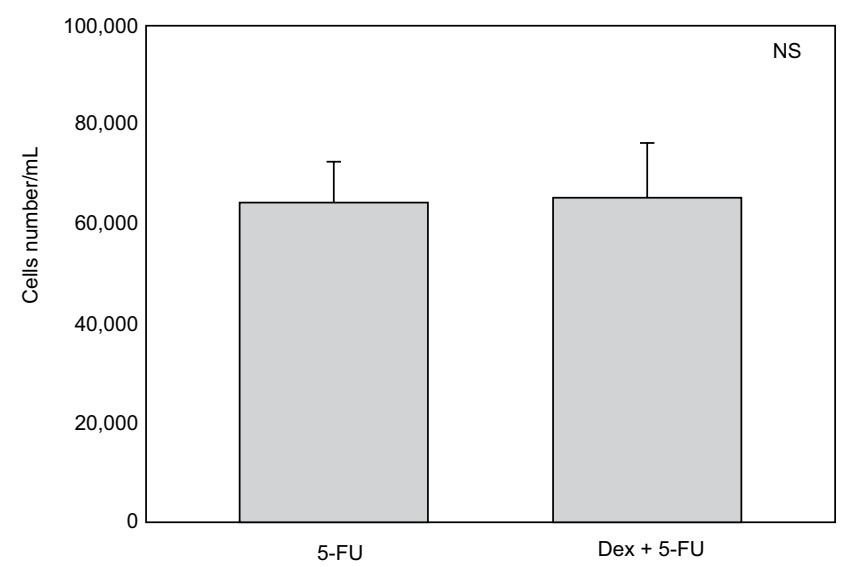

Figure 3 5-Fluorouracil (5-FU) with dexamethasone (Dex) pretreatment. MCF-7 cells were pretreated with $100 \mathrm{nM}$ of Dex prior to administration of $15 \mu \mathrm{M}$ of 5-FU. The graph indicates the number of cells $/ \mathrm{mL} 3$ days after treatment. No significant difference was discernible between the samples treated with Dex alone or Dex in combination with 5-FU $(n=4)$. Controls not shown (as for Figure I). Error bars indicate standard deviation.

Abbreviation: NS, nonsignificant.

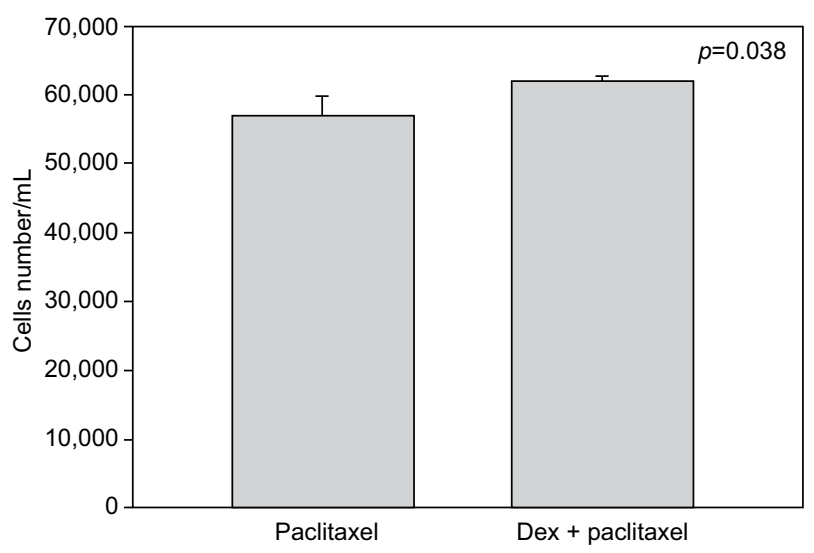

Figure 4 Paclitaxel with dexamethasone (Dex) pretreatment. MCF-7 cells were pretreated with $100 \mathrm{nM}$ of Dex prior to administration of $15 \mathrm{nM}$ of paclitaxel. The graph indicates the number of cells $/ \mathrm{mL} 3$ days after treatment. A significant difference was discernible between the samples treated with Dex alone or Dex in combination with paclitaxel $(n=4)$. Error bars indicate standard deviation.

action, we did not perform additional testing with doxorubicin. In this study, we demonstrated that the apoptotic activity of paclitaxel was significantly reduced (by $8.5 \%$ ) when MCF-7 cells were preexposed to Dex. No significant effect of Dex was seen with exposure to the chemotherapeutic agent 5-FU or epirubicin. Under in vitro conditions, paclitaxel enhances the polymerization of tubulin so as to form stable microtubules. It also interacts directly with microtubules and protects them from depolymerization by cold or calcium, both of which can readily depolymerize microtubules in the absence of the drug. Paclitaxel arrests cells at the G2-M phase of the cell cycle by the stabilization of spindle microtubules. However, the exact mechanism by which paclitaxel induces apoptosis is not clear. ${ }^{20} \mathrm{An}$ explanation could be that taxanes act more than docetaxel 
by phosphorylation of bcl2, thus abrogating the normal antiapoptotic function of bcl2. ${ }^{21}$

Wu et al ${ }^{17,18}$ demonstrated that pretreatment of breast cancer cell lines by Dex inhibits chemotherapy-induced apoptosis in a GR-dependent manner and that it is associated with transcriptional induction of at least two genes identified in our screening - SGK-1 and MKP-1.

By contrast, there was no difference in cell proliferation following treatment with 5-FU and epirubicin. Their mechanisms of action, however, are different from that of paclitaxel. 5-FU needs to be converted at the nucleotide level in order to exert its effect. It can be incorporated into RNA, leading to interference with the maturation of nuclear RNA. Its conversion to 5-fluoro-2'deoxy-5'monophosphate, which leads to inhibition of thymidylate synthase and subsequently DNA synthesis, is however considered to be its main mechanism of action..$^{15,22}$ Epirubicin is an anthracycline antibiotic; its antitumor activity is caused by its ability to interfere with transcription, resulting in inhibition of RNA synthesis. ${ }^{23}$

Under in vitro conditions, several, at times antagonistic, actions of GCs have been described..$^{2,3,8-14}$ This underscores the relevance of a direct study of preexposure to GCs prior to chemotherapy. In our study, the results were dependent on the specific chemotherapeutic agent being administered, which may be explained by the different cytotoxic mechanisms of these chemotherapeutics. However, the result of this study is not sufficient to warrant changing the current therapeutic approach. Other factors most certainly influence the tumor response in vivo. Indeed, it has been proposed that the peritumoral stroma and cancer-associated fibroblasts play an important role in breast tumorigenesis. ${ }^{24}$ The observed decrease in the efficacy of paclitaxel on MCF-7 cells previously exposed to Dex is, however, not sufficient to warrant changing the current therapeutic approach. Indeed, it has been proposed that the peritumoral stroma and cancer-associated fibroblasts play an important role in breast tumorigenesis. ${ }^{17,24}$ For example, in tumors that exhibit an aggressive metastatic phenotype, such as luminal HER2-positive and triple-negative, metalloproteinase-2 (MMP-2) expression is significantly increased. ${ }^{25}$ Dex could be acting at this level, by alleviating the effect of MMP-2. ${ }^{19,26}$ More recently, we demonstrated frequent expression of GRs in breast cancer-associated fibroblasts. The presence of GRs within breast tumors and the adjacent peritumoral stroma must be taken into account when GCs are prescribed to cancer patients. As shown in this study, GCs could substantially decrease the cellular response(s) caused by exposure to chemotherapeutics. The use of GCs other than Dex could be a solution. Recently, Orqueda et al showed that the rigid steroid 21-hydroxy-6,19-epoxyprogesterone
(21OH-6, 19OP), a selective GR ligand, behaves as a dissociated GC that retains anti-inflammatory action without affecting the apoptotic process triggered by chemotherapeutic drugs. Indeed, contrary to Dex, 21OH-6, 19OP neither reverts the paclitaxel-induced caspase-3 activity nor induces the antiapoptotic $\mathrm{Bcl}-\mathrm{X}(\mathrm{L})$ gene expression in murine tumor mammary epithelial cells. ${ }^{27}$

\section{Conclusion}

Since GCs affect the behavior of numerous cancer cell types, their function should be defined more precisely if they are to be used in conjunction with chemotherapy. Indeed, in this study we demonstrated a decrease in the response of MCF-7 cells to paclitaxel when they had been pretreated with Dex. Use of other antiemetogenic molecules should be considered in light of these results.

\section{Disclosure}

The authors report no conflicts of interest in this work.

\section{References}

1. Rosen J, Miner JN. The search for safer glucocorticoid receptor ligands. Endocr Rev. 2005;26(3):452-464.

2. Kofler R. The molecular basis of glucocorticoid-induced apoptosis of lymphoblastic leukemia cells. Histochem Cell Biol. 2000;114(1):1-7.

3. Evans-Storms RB, Cidlowski JA. Delineation of an antiapoptotic action of glucocorticoids in hepatoma cells: the role of nuclear factor-kappaB. Endocrinology. 2000;141(5):1854-1862.

4. Bernard-Marty C, Cardoso F, Piccart MJ. Use and abuse of taxanes in the management of metastatic breast cancer. Eur J Cancer. 2003; 39(14):1978-1989.

5. Micha JP, Rettenmaier MA, Brown JV 3rd, et al. A randomized controlled pilot study comparing the impact of aprepitant and fosaprepitant on chemotherapy induced nausea and vomiting in patients treated for gynecologic cancer. Int J Gynecol Cancer. 2016;26(2): 389-393.

6. Coates AS, Winer EP, Goldhirsch A, et al. Tailoring therapies - improving the management of early breast cancer: St Gallen International Expert Consensus on the Primary Therapy of Early Breast Cancer 2015. Ann Oncol. 2015;26(8):1533-1546.

7. Senkus E, Kyriakides S, Ohno S, et al. Primary breast cancer: ESMO Clinical Practice Guidelines for diagnosis, treatment and follow-up. Ann Oncol. 2015;26(Suppl 5):v8-v30.

8. Skor MN, Wonder EL, Kocherginsky M, et al. Glucocorticoid receptor antagonism as a novel therapy for triple-negative breast cancer. Clin Cancer Res. 2013;19(22):6163-6172.

9. Lien HC, Lu YS, Cheng AL, et al. Differential expression of glucocorticoid receptor in human breast tissues and related neoplasms. J Pathol. 2006;209(3):317-327.

10. Buxant F, Engohan-Aloghe C, Noël JC. Estrogen receptor, progesterone receptor, and glucocorticoid receptor expression in normal breast tissue, breast in situ carcinoma, and invasive breast cancer. Appl Immunohistochem Mol Morphol. 2010;18(3):254-257.

11. Vilasco M, Communal L, Hugon-Rodin J, et al. Loss of glucocorticoid receptor activation is a hallmark of BRCA1-mutated breast tissue. Breast Cancer Res Treat. 2013;142(2):283-296.

12. Buxant F, Kindt N, Laurent G, Noël JC, Saussez S. Antiproliferative effect of dexamethasone in the MCF-7 breast cancer cell line. Mol Med Rep. 2015;12(3):4051-4054. 
13. Lippman M, Bolan G, Huff K. The effects of glucocorticoids and progesterone on hormone-responsive human breast cancer in long-term tissue culture. Cancer Res. 1976;36(12):4602-4609.

14. Leo JC, Guo C, Woon CT, Aw SE, Lin VC. Glucocorticoid and mineralocorticoid cross talk with progesterone receptor to induce focal adhesion and growth inhibition in breast cancer cells. Endocrinology. 2004;145(3):1314-1321.

15. Khan S, Lopez-Dee Z, Kumar R, Ling J. Activation of NFkB is a novel mechanism of pro-survival activity of glucocorticoids in breast cancer cells. Cancer Lett. 2013;337(1):90-95.

16. Schorr K, Furth PA. Induction of bcl-xL expression in mammary epithelial cells is glucocorticoid-dependent but not signal transducer and activator of transcription 5-dependent. Cancer Res. 2000; 60(21):5950-5953.

17. Wu W, Chaudhuri S, Brickley DR, Pang D, Karrison T, Conzen SD. Microarray analysis reveals glucocorticoid-regulated survival genes that are associated with inhibition of apoptosis in breast epithelial cells. Cancer Res. 2004;64(5):1757-1764.

18. Wu W, Pew T, Zou M, Pang D, Conzen SD. Glucocorticoid receptorinduced MAPK phosphatase-1 (MPK-1) expression inhibits paclitaxelassociated MAPK activation and contributes to breast cancer cell survival. J Biol Chem. 2005;280(6):4117-4124.

19. Rutz HP. Effects of corticosteroid use on treatment of solid tumours. Lancet. 2002;360(9349):1969-1970.
20. Gotaskie GE, Andreassi BF. Paclitaxel: a new antimitotic chemotherapeutic agent. Cancer Pract. 1994;2(1):27-33.

21. Haldar S, Basu A, Croce CM. Bcl2 is the guardian of microtubule integrity. Cancer Res. 1997;57(2):229-233.

22. Pinedo HM, Peters GF. Fluorouracil: biochemistry and pharmacology. J Clin Oncol. 1988;6(10):1653-1664.

23. Wang F, Liu X, Liu C, Liu Z, Sun L. Effects of antibiotic antitumor drugs on nucleotide levels in cultured tumor cells: an exploratory method to distinguish the mechanisms of antitumor drug action based on targeted metabolomics. Acta Pharm Sin B. 2015;5(3):223-230.

24. Luo H, Tu G, Liu Z, Liu M. Cancer-associated fibroblasts: a multifaceted driver of breast cancer progression. Cancer Lett. 2015;361(2): 155-163.

25. Catteau X, Simon P, Noël JC. Stromal expression of matrix metalloproteinase 2 in cancer-associated fibroblasts is strongly related to human epidermal growth factor receptor 2 status in invasive breast carcinoma. Mol Clin Oncol. 2016;4(3):375-378.

26. Wang J, Zhang $\mathrm{H}, \mathrm{Su} \mathrm{C}$, et al. Dexamethasone ameliorates $\mathrm{H}_{2} \mathrm{~S}$-induced acute lung injury by alleviating matrix metalloproteinase-2 and -9 expression. PLoS One. 2014;9(4):e94701.

27. Orqueda AJ, Dansey MV, Español A, et al. The rigid steroid 21-hydroxy6,19-epoxyprogesterone (21OH-6,19OP) is a dissociated glucocorticoid receptor modulator potentially useful as a novel coadjuvant in breast cancer chemotherapy. Biochem Pharmacol. 2014;89(4):526-535.
Breast Cancer - Targets and Therapy

\section{Publish your work in this journal}

Breast Cancer - Targets and Therapy is an international, peerreviewed open access journal focusing on breast cancer research, identification of therapeutic targets and the optimal use of preventative and integrated treatment interventions to achieve improved outcomes, enhanced survival and quality of life for the cancer patient

\section{Dovepress}

The manuscript management system is completely online and includes a very quick and fair peer-review system, which is all easy to use. Visit http://www.dovepress.com/testimonials.php to read real quotes from published authors. 